\title{
Association Between Social Phobia and Parenting Styles Among Secondary School Students
}

\author{
Eman Shokry Abdallah ${ }^{1}$, Hanaa Hamdy ELzeiny ${ }^{2}$, Rehab Fathy Abdel-hady², \\ Marwa Samy El-Sheikh ${ }^{2}$ \\ ${ }^{1}$ Community Health Nursing Dep., Faculty of Nursing, Zagazig University, Zagazig City, Egypt \\ ${ }^{2}$ Psychiatric and Mental Health Nursing Dep., Faculty of Nursing, Zagazig University, Zagazig City, Egypt
}

Email address:

Emanshokry2012@yahoo.com (E. S. Abdallah)

\section{To cite this article:}

Eman Shokry Abdallah, Hanaa Hamdy ELzeiny, Rehab Fathy Abdel-hady, Marwa Samy El-Sheikh. Association Between Social Phobia and Parenting Styles Among Secondary School Students. American Journal of Nursing Science. Vol. 5, No. 3, 2016, pp. 96-105.

doi: 10.11648/j.ajns.20160503.14

Received: April 7, 2016; Accepted: May 3, 2016; Published: May 14, 2016

\begin{abstract}
Social phobia (SP) is one of the most prevalent anxiety disorders among adolescent youth and remains under recognized and under treated. Environmental factors specifically parenting styles are particularly significant in the development of social phobia. Aim of the present study was to determine the association between social phobia and parenting styles among secondary school students. A descriptive design was selected in carrying out this study. The Setting of the present study was in the governmental schools in ElSinbellawin city, Dakhlia governerate. The sample size included a simple random sample composed of 446 students from three secondary schools; Ahmed Lofty El-Sayed secondary school for boys, El-Sadat secondary school for boys and The Secondary school for girls. Tools used for data collection were; sociodemographic data sheet, parenting styles and social phobia scales. Results revealed that female students recorded higher prevalence of social phobia (23.6\%) than did in male students (14.4\%). The significant difference was revealed between authoritarian, neglectful parenting styles and developing social phobia in the female students. There was a significant negative correlation between parenting styles and social phobia. This study concluded that social phobia is higher in females than in males. More the parents showed (authoritarian, authoritative, over-protective, neglectful) parenting style, their children showed lower levels of social phobia. The study recommended with early identification of socio phobic students by school nurse and social worker will be successfully help in providing early and adequate treatment, which in turn, reduce the burden of this common condition. Providing parents with educational programs about the efficacy of authoritative parenting style in nurturing their children.
\end{abstract}

Keywords: Social Phobia, Parenting Styles, Adolescence Age

\section{Introduction}

Social phobia (SP), also called social anxiety disorder (SAD), is a chronic and disabling conditioned [1] It is characterized by marked or intense fear of social or performance-based situations where scrutiny or evaluation by others may occur. This scrutiny may be the result of social interactions (e.g., having a conversation), being observed (e.g., eating or working), or performing in front of others (e.g., public speaking) [2]. According to the fourth edition of the Diagnostic and Statistical Manual of Mental Disorders (DSM-IV-TR), subtypes of social phobia have been recognized including generalized social phobia which is a fear of most social interactions combined with fear of most performance situations, and non-generalized (circumscribed, or specific) social phobia in which persons are afraid of only one type of performance situation or afraid of only a few rather than most social situations [3]. However, The DSM-5 replaced the "generalized" subtype of social phobia with "performance only" that identify those individuals whose "fear is restricted to speaking or performing in public" [4]. Social phobia typically occurs in early to middle adolescence between 10 to 17 years of age [5], with a high prevalence rate between $10 \%$ and $15 \%$ [6]. It is worth noting that social phobia is being as twice among girls as among boys. [7]. Social phobia is correlated with_significant functional 
impairment in educational achievement, occupational, performance, social interaction, relationships, and quality of life [8]. Parenting styles has been particularly emphasized as a putative risk factor for offspring social phobi [9]. Parenting styles was developed by Psychologist, Diana Baumrind that were identified as psychological constructs which represent standard strategies parents use in raising their children [10], including: Authoritative, Authoritarian and Permissive Parenting Style [11]. Psychologists Maccoby \& Martin identified a fourth parenting style, called uninvolved or neglectful styles of parenting [12]. As stated by Baumrind, children who are raised by authoritative parents tend to perform better in society as they exhibit higher levels of psychosocial competence and social development [13]. Regarding the role of psychiatric and mental health nurse is to help the student to identify automatic negative thoughts, provide them with competing responses to negative thoughts or behaviors, encourage students for positive self-talk and rehearse social skills in a smaller or more relaxed setting. Hence, providing a sympathetic and tolerant environment and making some adaptations may be helpful to aid adolescents with social phobia [14].

\subsection{Aim of the Study}

To determine the association between social phobia and parenting styles among secondary school students.

\subsection{Significance of Study}

Social phobia (SP) is among the most common mental disorders in children and adolescents [15]. It is considered as a serious mental health problem due to its high prevalence and the resulting impairments in performance and social interactions [16]. Parenting styles have a crucial impact on the psychological development of the adolescents and their future [17]. Any failure in the parents' role may induce unwanted damaging results on children's growth and may lead to misbehavior problems [18].

\section{Methodology and Methods}

\subsection{Research Question}

What is the association between social phobia and parenting styles among the secondary school students?

\subsection{Research Design}

Descriptive design was used in this study.

\subsection{Setting}

The study was conducted at three governmental secondary schools in Sinbellaween city (Ahmed Lofty El-Slayed secondary school for boys, El-Sadat secondary school for boys and the secondary school for girls).

\subsection{Sample}

The sample was calculated to be 446 students that will be selected from the previous mentioned settings according to inclusion criteria.

\subsection{Inclusion Criteria}

a-Ages from 15 to 17 years.

b- Both males and females.

Free from physical disability or chronic illness.-c

d- Agreement of students to participate in the study.

\subsection{Tools of Data Collection: Three Tools Were Used to Collect the Necessary Data as Follows}

Tool (I): Socio-demographic data sheet [19]: to assess the personal characteristic of the students and their parents. This scale composed of 7 domains, including Education domain of both father and mother ( 8 items), Occupation domain of both father and mother (6 items), Family domain (4 items), Family possessions domain (12 items), Economic domain (3 items), Home sanitation domain (3 items) and Health care domain (1 item).

Scoring system: The total score is (84) degree. To determine the socioeconomic class of the students through using this scale, the scoring system was calculated as follows: score equal or less than $42 \%$ would be considered as a low social class, score from $43 \%$ to less than $63 \%$ would be considered as middle social class, and score equal to or more than $64 \%$ would be considered as high social class.

Tool II. Parental Styles Scale [20]: it was used to measure the students' perceptions of their parents' parenting style. This scale is consisted of two parallel versions, with one for the mother and one for the father. Each version comprised 60 items which assessed the following four dimensions of parenting styles i.e. authoritative style, authoritarian style, over-protective and neglectful style. Assessment is measured on 4 point Likert Scale, The four categories for scoring system are: (1) Always, (2) Often, (3) Sometimes, (4) Never.

Dimension (1): Authoritative and authoritarian styles:

It includes two images, image (A) for father and image (B) for mother that consists of (30) sentences for each image, measuring parental responses as perceived by the children in a number of situations.

This scale has positive sentences no. $(3,5,7,11,13,18$, 21, 28, 29 and 30) that were scored as 4, 3, 2 and 1 for the responses

"Always", "Often", "Sometimes" and "never".

In addition, this scale has a negative sentence no. (1, 2, 4, $6,8,9,10,12,14,15,16,17,19,20,22,23,24,25,26$ and 27) that were scored 1, 2, 3 and 4 for the following responses "Always", "Often", "Sometimes" and "never".

Dimension (2): overprotective and neglectful styles: It includes two images: image (A) for father and image (B) for mother that consists of (30) sentences for each image, measuring parental responses as perceived by the children in a number of situations.

This scale has positive sentences no. $(31,32,33,34,35$, $36,37,38,40,41,42,43,44,45,46,47,48,50,53$ and 59) that were scored as 4, 3, 2 and 1 for the responses "Always", 
"Often", "Sometimes" and "never". Also, this scale have a negative sentences no. $(39,49,51,52,54,55,56,57,58$ and $60)$ that were scored as $1,2,3$ and 4 for the following responses "Always", "Often", "Sometimes" and "never"

Tool III. Social Phobia Scale [21]: it was translated by ElDesoky [22], it was constructed to assess social phobia of students. It consists of 23 items and assessment is measured on two -point Likert Scale: (1) yes, (2) No.

Scoring system

The Social phobia scale's items were respectively scored as following: Giving "2" grades for answering with "Yes" and "1" grade for answering with "No" except the sentences $(3,7$. $14,16,17,27,28)$ are scored in the opposite direction through giving "1" grade for answering with "Yes" and "2" grades for answering with "No".

\subsection{Pilot Study}

A pilot study was conducted on 40 students from the two schoolar stages, completed by the students themselves. It was done to test the feasibility and clarity of the tools, and also helped to know the time needed for filling the tools and it didn't need any modification and they had been excluded later in the study.

\subsection{Administrative Design}

After approval of the ethics committee, the permission of conducting the study was obtained by submission of an official letter issued from the dean of the faculty of nursing at Zagazig University to the Security Department in the Directorate of Education at Mansoura city, after that to directors of Administration in El-Sinbellawin city. The researcher visited these three schools, met with the Administrators of schools, explained to them the study aim and the importance of the study and procedures and asked for their cooperation to conduct the study and facilitate data collection.

\subsection{Ethical Considerations}

The students were given a verbal description of the aim of the study, the benefits, and nonparticipation or withdrawal rights at any time without giving any reason. Additionally, they were informed that their participation in this study is voluntary, no names were included in the questionnaire sheet and anonymity of each participant was protected by the allocation of code number for each student. The researcher stressed on a confidentiality of the gathered information and will be used only for the purpose of the study.

\subsection{Statistical Design}

Data entry and statistical analysis were done using (SPSS) version 14.0 (Statistical Package for Social Sciences). Data were presented using descriptive statistics in the form of frequencies and percentages for qualitative variables, and means and standard deviations for quantitative variables. Qualitative categorical variables were compared using a chisquare test. Whenever the expected values in one or more of the cells in a $2 \times 2$ table were less than 5 , Fisher exact test was used instead. Pearson correlation analysis was used for assessment of the interrelationships among quantitative variables, and Spearman rank correlation for ranking ones. Pvalue was statistical significance at $<0.05$, and high statistical significance at $<0.01$. The given graphs were constructed using Microsoft Excel Software.

\section{Result}

Table 1 shows Socio- demographic characteristics of the studied students and their parents. Shows that the mean age of the studied students was $16 \pm 1$ years and $(53 \%)$ of both males and females students were at age of 16 years. Regarding, the scholastic grade of the studied students, more than half of both males' students $(53.1 \%)$ and females students $(65.1 \%)$ were from the $1^{\text {st }}$ grade.

Table 1. Socio- demographic characteristics of the studied students $(n=446)$.

\begin{tabular}{|c|c|c|c|c|}
\hline \multirow{3}{*}{ Sociodemographic data } & \multicolumn{4}{|c|}{ Gender } \\
\hline & \multicolumn{2}{|c|}{ Male $(n=209)$} & \multicolumn{2}{|c|}{ Female $(n=237)$} \\
\hline & No & $\%$ & No & $\%$ \\
\hline \multicolumn{5}{|l|}{ Age: } \\
\hline - 15 & 23 & 11.0 & 60 & 25.3 \\
\hline - 16 & 112 & 53.6 & 127 & 53.6 \\
\hline - 17 & 74 & 35.4 & 50 & 21.1 \\
\hline - Mean+SD & $16 \pm 1$ & & $16 \pm 1$ & \\
\hline \multicolumn{5}{|l|}{ Grade: } \\
\hline - 1 & 111 & 53.1 & 133 & 56.1 \\
\hline - 2 & 98 & 46.9 & 104 & 43.9 \\
\hline \multicolumn{5}{|l|}{ Marital status of parents: } \\
\hline - Married & 195 & 93.3 & 215 & 90.7 \\
\hline - Divorced & 14 & 6.7 & 11 & 4.6 \\
\hline - widowed & 0 & 0.0 & 11 & 4.6 \\
\hline \multicolumn{5}{|l|}{ educational level of father: } \\
\hline - Illiterate & 7 & 3.3 & 7 & 3.0 \\
\hline - Read \& write & 19 & 9.1 & 19 & 8.0 \\
\hline - Primary & 10 & 4.8 & 10 & 4.2 \\
\hline - Preparatory & 16 & 7.7 & 11 & 4.6 \\
\hline - Secondary & 40 & 19.1 & 64 & 27.0 \\
\hline - Intermediate (2years) institutes & 14 & 6.7 & 26 & 11.0 \\
\hline - University graduate & 82 & 39.2 & 86 & 36.3 \\
\hline - Postgraduate degree & 21 & 10.0 & 14 & 5.9 \\
\hline \multicolumn{5}{|l|}{ educational level of mother: } \\
\hline - Illiterate & 13 & 6.2 & 11 & 4.6 \\
\hline - Read \& write & 18 & 8.6 & 7 & 3.0 \\
\hline - Primary & 5 & 2.4 & 6 & 2.5 \\
\hline - Preparatory & 11 & 5.3 & 7 & 3.0 \\
\hline - Secondary & 53 & 25.4 & 94 & 39.7 \\
\hline - Intermediate (2 years) institutes & 16 & 7.7 & 30 & 12.7 \\
\hline - University graduate & 73 & 34.9 & 77 & 32.5 \\
\hline - Postgraduate degree & 20 & 9.6 & 5 & 2.1 \\
\hline \multicolumn{5}{|l|}{ Residence: } \\
\hline - Urban slum & 1 & 0.05 & 1 & 0.4 \\
\hline - Rural & 89 & 42.6 & 119 & 50.2 \\
\hline - urban & 119 & 56.9 & 117 & 49.4 \\
\hline \multicolumn{5}{|l|}{ Socioeconomic level } \\
\hline - high level & 57 & 27.3 & 48 & 20.3 \\
\hline - moderate level & 116 & 55.5 & 153 & 64.6 \\
\hline - low level & 36 & 17.2 & 36 & 15.2 \\
\hline
\end{tabular}

Relation between social phobia and sociodemographic characteristics of the studied male students were illustrated in Table 2. With regard to parents' marital status, social phobia 
was recorded as $(35.7 \%)$ of male students for divorced parents compared to none in males students for widowed parents and $(12.8 \%)$ of males for married parents, with statistically significant difference $(\mathrm{P}=0.018)$. As for residence, social phobia was found in all males students $(100 \%)$ who live at urban slum areas compared to $(18.0 \%)$ of students who live at rural areas and $(10.9 \%)$ of those who are from urban areas with statistically significant difference $(\mathrm{P}=0.018)$.

Table 2. Relation between social phobia and sociodemographic characteristics among the studied male students.

\begin{tabular}{|c|c|c|c|c|c|}
\hline \multirow{3}{*}{$\begin{array}{l}\text { Sociodemographic } \\
\text { variables }\end{array}$} & \multicolumn{4}{|c|}{ Social phobia among males $(n=209)$} & \multirow{3}{*}{ МCP } \\
\hline & \multicolumn{2}{|l|}{ No } & \multicolumn{2}{|l|}{ Yes } & \\
\hline & No & $\%$ & No & $\%$ & \\
\hline \multicolumn{6}{|l|}{ Age: } \\
\hline - 15 & 20 & 87.0 & 3 & 13.0 & \multirow{3}{*}{0.934} \\
\hline - 16 & 95 & 84.8 & 17 & 15.2 & \\
\hline - 17 & 64 & 86.5 & 10 & 13.5 & \\
\hline \multicolumn{6}{|l|}{ Secondary grade: } \\
\hline - $1^{\mathrm{st}}$ & 96 & 86.5 & 15 & 13.5 & \multirow[t]{2}{*}{$0.712 !$} \\
\hline - $2^{\text {nd }}$ & 83 & 84.7 & 15 & 15.3 & \\
\hline \multicolumn{6}{|l|}{$\begin{array}{l}\text { Marital status of the } \\
\text { parents: }\end{array}$} \\
\hline - Married & 170 & 87.2 & 25 & 12.8 & \multirow[t]{3}{*}{0.018 ! } \\
\hline - Divorced & 9 & 64.3 & 5 & 35.7 & \\
\hline - Widowed & 0 & 0.0 & 0 & 0.0 & \\
\hline \multicolumn{6}{|l|}{ Residence: } \\
\hline - Urban slum & 0 & 0.0 & 1 & 100.0 & \multirow{3}{*}{0.018 ! } \\
\hline - Rural & 73 & 82.0 & 16 & 18.0 & \\
\hline - Urban & 106 & 89.1 & 13 & 10.9 & \\
\hline \multicolumn{6}{|l|}{ Socioeconomic level: } \\
\hline - Moderate level & $\begin{array}{l}49 \\
98\end{array}$ & $\begin{array}{l}86.0 \\
84.5\end{array}$ & $\begin{array}{l}8 \\
18\end{array}$ & $\begin{array}{l}14.0 \\
15.5\end{array}$ & \multirow[t]{2}{*}{0.802} \\
\hline - Low level & 32 & 88.9 & 4 & 11.1 & \\
\hline
\end{tabular}

Table 3 shows that there was no statistical significant difference between social phobia in male students and their parents' social characters.

Table 3. Relation between social phobia in male students and their parents social characters.

\begin{tabular}{|c|c|c|c|c|c|}
\hline \multirow{3}{*}{ Parents' Social Characters } & \multicolumn{4}{|c|}{$\begin{array}{l}\text { Social phobia among males } \\
(n=209)\end{array}$} & \multirow[t]{3}{*}{ MCP } \\
\hline & \multicolumn{2}{|l|}{ YES } & \multicolumn{2}{|l|}{ NO } & \\
\hline & No. & $\%$ & No. & $\%$ & \\
\hline \multicolumn{6}{|l|}{ Educational level of father: } \\
\hline - Illiterate & 7 & 100.0 & 0 & 0.0 & \multirow{8}{*}{0.308} \\
\hline - Read\& write & 17 & 89.5 & 2 & 10.5 & \\
\hline - Primary & 10 & 100.0 & 0 & 0.0 & \\
\hline - Preparatory & 11 & 68.8 & 5 & 31.3 & \\
\hline - Secondary & 34 & 85.0 & 6 & 15.0 & \\
\hline $\begin{array}{l}\text { - Intermediate ( } 2 \text { years } \\
\text { institute) }\end{array}$ & 13 & 92.9 & 1 & 7.1 & \\
\hline - University graduate & 68 & 82.9 & 14 & 17.1 & \\
\hline - Postgraduate degree & 19 & 90.5 & 2 & 9.5 & \\
\hline \multicolumn{6}{|l|}{ Educational level of mother: } \\
\hline - Illiterate & 10 & 76.9 & 3 & 23.1 & \multirow{8}{*}{0.344} \\
\hline - Read\& write & 17 & 94.4 & 1 & 5.6 & \\
\hline - Primary & 4 & $8-.0$ & 1 & 20.0 & \\
\hline - Preparatory & 11 & 100.0 & 0 & 0.0 & \\
\hline - Secondary & 44 & 83.0 & 9 & 17.0 & \\
\hline $\begin{array}{l}\text { - Intermediate ( } 2 \text { years } \\
\text { institute) }\end{array}$ & 15 & 93.8 & 1 & 6.3 & \\
\hline - University graduate & 59 & 80.8 & 14 & 19.2 & \\
\hline - Postgraduate degree & 19 & 95.0 & 1 & 5.0 & \\
\hline
\end{tabular}

\begin{tabular}{|c|c|c|c|c|c|}
\hline \multirow{3}{*}{ Parents' Social Characters } & \multicolumn{4}{|c|}{$\begin{array}{l}\text { Social phobia among males } \\
(\mathrm{n}=209)\end{array}$} & \multirow[t]{3}{*}{ MCP } \\
\hline & \multicolumn{2}{|c|}{ YES } & \multicolumn{2}{|c|}{ NO } & \\
\hline & No. & $\%$ & No. & $\%$ & \\
\hline \multicolumn{6}{|l|}{ Father profession: } \\
\hline - Non-working & 1 & 100.0 & 0 & 0.0 & \multirow{6}{*}{0.978} \\
\hline - Unskilled manual worker & 24 & 82.8 & 5 & 17.2 & \\
\hline $\begin{array}{l}\text { - Skilled manual } \\
\text { worker/farmer }\end{array}$ & 19 & 90.5 & 2 & 9.5 & \\
\hline - Trades/business & 25 & 86.2 & 4 & 13.8 & \\
\hline - Semi-professional/clerk & 69 & 85.2 & 12 & 14.8 & \\
\hline - Professional & 41 & 85.4 & 7 & 14.6 & \\
\hline \multicolumn{6}{|l|}{ Mother Profession: } \\
\hline - Non-working & 97 & 85.8 & 16 & 14.2 & \multirow{6}{*}{0.914} \\
\hline - Unskilled manual worker & 5 & 100.0 & 0 & 0.0 & \\
\hline $\begin{array}{l}\text { - Skilled manual } \\
\text { worker/farmer }\end{array}$ & 1 & 100.0 & 0 & 0.0 & \\
\hline - Trades/business & 1 & 100.0 & 0 & 0.0 & \\
\hline - Semi-professional/clerk & 55 & 83.3 & 11 & 16.7 & \\
\hline - Professional & 20 & 87.0 & 3 & 13.0 & \\
\hline \multicolumn{6}{|l|}{ Family income: } \\
\hline - Indebt & 9 & 90.0 & 1 & 10 & \multirow{4}{*}{0.806} \\
\hline - Just meet routine expenses & 3.4 & 87.2 & 5 & 12.8 & \\
\hline $\begin{array}{l}\text { - Meet routine expenses and } \\
\text { emergencies }\end{array}$ & 67 & 82.7 & 14 & 17.3 & \\
\hline - Able to save /invest money & 69 & 87.3 & 10 & 12.7 & \\
\hline
\end{tabular}

Relation between social phobia and sociodemographic characteristics among the studied female students were clarified in Table (4), regarding parents' marital status, social phobia was recorded in $(36.4 \%)$ of females students for divorced and widowed parents compared to $(22.3 \%)$ of females for married parents, with statistically insignificant difference $(\mathrm{P}=0.336$. As for residence, social phobia was found in $(24.4 \%)$ of females students who live in rural areas and $(23.1 \%)$ of females who live at urban areas compared to none in females from urban slum areas with statistically insignificant difference $(\mathrm{P}=0.833)$.

Table 4. Relation between social phobia and sociodemographic characteristics among the studied female students.

\begin{tabular}{|c|c|c|c|c|c|}
\hline \multirow{3}{*}{$\begin{array}{l}\text { Sociodemographic } \\
\text { variables }\end{array}$} & \multicolumn{4}{|c|}{ Social phobia among females $(n=237)$} & \multirow{3}{*}{ МCP } \\
\hline & \multirow{2}{*}{$\begin{array}{l}\text { No } \\
\text { No } \\
\end{array}$} & & \multicolumn{2}{|c|}{ Yes } & \\
\hline & & $\%$ & No & $\%$ & \\
\hline \multicolumn{5}{|l|}{ Age: } & \multirow{4}{*}{0.809} \\
\hline - 15 & 44 & 73.3 & 16 & 26.7 & \\
\hline - 16 & 98 & 77.2 & 29 & 22.8 & \\
\hline - 17 & 39 & 78.0 & 11 & 22.0 & \\
\hline \multicolumn{5}{|l|}{ Secondary grade: } & \multirow{3}{*}{0.628} \\
\hline - $1^{\text {st }}$ & 100 & 75.2 & 33 & 24.8 & \\
\hline - $2^{\text {nd }}$ & 81 & 77.9 & 23 & 22.1 & \\
\hline \multicolumn{5}{|l|}{$\begin{array}{l}\text { Marital status of the } \\
\text { parents: }\end{array}$} & \multirow{4}{*}{0.336} \\
\hline - Married & 167 & 77.7 & 48 & 22.3 & \\
\hline - Divorced & 7 & 63.6 & 4 & 36.4 & \\
\hline - Widowed & 7 & 63.6 & 4 & 36.4 & \\
\hline \multicolumn{5}{|l|}{ Residence: } & \multirow{4}{*}{0.833} \\
\hline - Urban slum & 1 & 100.0 & 0 & 0.0 & \\
\hline - Rural & 90 & 75.6 & 29 & 24.4 & \\
\hline - Urban & 90 & 75.9 & 27 & 23.1 & \\
\hline \multicolumn{5}{|l|}{ Socioeconomic level: } & \multirow{4}{*}{0.131} \\
\hline - High level & 34 & 70.8 & 14 & 29.2 & \\
\hline - Moderate level & 123 & 80.4 & 30 & 19.6 & \\
\hline - Low level & 24 & 66.7 & 12 & 33.3 & \\
\hline
\end{tabular}


Relation between social phobia in female students and their parents' social characters were revealed in Table 5., social phobia was highest recorded $(83.3 \%)$ among female students with a primary educated mothers, among whose mothers were unskilled manual workers $(80.0 \%)$ and whose family income just meet routine expenses (39.2\%) with statistically significant difference $(p=0.01,0.05,0.02$ respectively).

Table 5. Relation between social phobia in female students and their parents' social characters.

\begin{tabular}{|c|c|c|c|c|c|}
\hline \multirow{3}{*}{ Parents' Social Characters } & \multicolumn{4}{|c|}{$\begin{array}{l}\text { Social phobia among } \\
\text { females }(n=237)\end{array}$} & \multirow[t]{3}{*}{ МCP } \\
\hline & \multicolumn{2}{|l|}{ YES } & \multicolumn{2}{|l|}{ NO } & \\
\hline & No. & $\%$ & No. & $\%$ & \\
\hline \multicolumn{5}{|l|}{ Educational level of father: } & \multirow{9}{*}{0.599} \\
\hline - Illiterate & 4 & 57.1 & 3 & 42.9 & \\
\hline - Read\& write & 15 & 78.9 & 4 & 21.1 & \\
\hline - Primary & 6 & 60.0 & 4 & 40.0 & \\
\hline - Preparatory & 8 & 72.7 & 3 & 27.3 & \\
\hline - Secondary & 50 & 78.1 & 14 & 21.9 & \\
\hline - Intermediate (2 years institute) & 23 & 88.5 & 3 & 11.5 & \\
\hline - University graduate & 65 & 75.6 & 21 & 24.4 & \\
\hline - Postgraduate degree & 10 & 71.4 & 4 & 28.6 & \\
\hline \multicolumn{5}{|l|}{ Educational level of mother: } & \multirow{9}{*}{$0.013^{*}$} \\
\hline - Illiterate & 8 & 72.7 & 3 & 27.3 & \\
\hline - Read\& write & 4 & 57.1 & 3 & 42.9 & \\
\hline - Primary & 1 & 16.7 & 5 & 83.3 & \\
\hline - Preparatory & 5 & 71.4 & 2 & 28.6 & \\
\hline - Secondary & 80 & 85.1 & 14 & 14.9 & \\
\hline - Intermediate (2 years institute) & 22 & 73.3 & 8 & 26.7 & \\
\hline - University graduate & 57 & 74.0 & 20 & 26.0 & \\
\hline - Postgraduate degree & 4 & 80.0 & 1 & 20.0 & \\
\hline \multicolumn{5}{|l|}{ Father profession: } & \multirow{7}{*}{0.508} \\
\hline - Non-working & 2 & 66.7 & 1 & 33.3 & \\
\hline - Unskilled manual worker & 22 & 81.5 & 5 & 18.5 & \\
\hline - Skilled manual worker/farmer & 25 & 67.6 & 12 & 32.4 & \\
\hline - Trades/business & 12 & 75.0 & 4 & 25.0 & \\
\hline - Semi-professional/clerk & 80 & 74.8 & 27 & 25.2 & \\
\hline - professional & 40 & 85.1 & 7 & 17.9 & \\
\hline \multicolumn{5}{|l|}{ Mother Profession: } & \multirow{7}{*}{$0.050 *$} \\
\hline - Non-working & 112 & 79.4 & 29 & 20.6 & \\
\hline - Unskilled manual worker & 1 & 20.0 & 4 & 80.0 & \\
\hline - Skilled manual worker/farmer & 1 & 50.0 & 1 & 50.0 & \\
\hline - Trades/business & 2 & 100.0 & 0 & 0.0 & \\
\hline - Semi-professional/clerk & 37 & 74.0 & 13 & 26.0 & \\
\hline - Professional & 28 & 75.7 & 9 & 24.3 & \\
\hline \multicolumn{5}{|l|}{ Family income: } & \multirow{5}{*}{$0.026^{*}$} \\
\hline - Indebt & 14 & 87.5 & 2 & 12.5 & \\
\hline \multirow{3}{*}{$\begin{array}{l}\text { - Just meet routine expenses } \\
\text { - Meet routine expenses and } \\
\text { emergencies } \\
\text { - Able to save /invest money }\end{array}$} & 31 & 60.8 & 20 & 39.2 & \\
\hline & 72 & 79.1 & 19 & 20.9 & \\
\hline & 64 & 81.0 & 15 & 19.0 & \\
\hline
\end{tabular}

Figure 1 displays that the prevalence of social phobia was significantly higher among females $(23.6 \%)$ than among males $(14.4 \%)$.

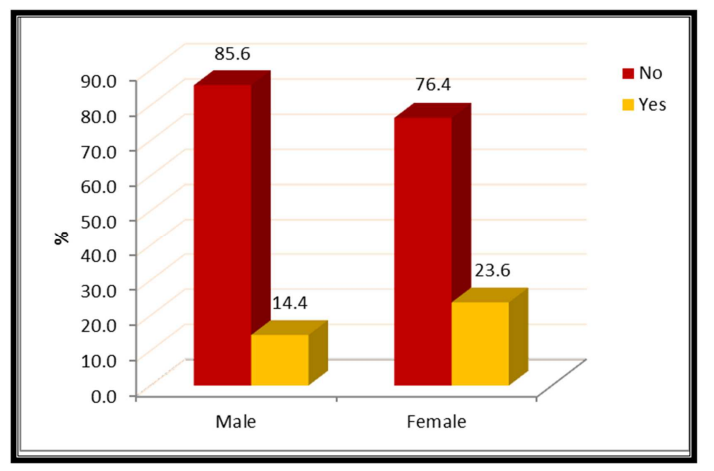

Figure 1. Comparison between male and female students regarding prevalence of social phobia.

Table 6 shows that, regarding the first domain of father's parenting styles; females in comparison with males had more paternal authoritative style, with $(78.5 \%)$ in females versus $(70.8 \%)$ in males, whereas males in comparison with females had more paternal authoritarian style, with $(26.8 \%)$ in males versus $(19.4 \%)$ in females with statistically insignificant difference $(\mathrm{P}=0.196)$. As regards the second domain of father's parenting styles, the same table also shows that females in comparison with males had more paternal overprotective style, with $(67.9 \%)$ in females versus $(64.1 \%)$ in males, whereas males in comparison with females had more paternal neglectful style, with $(32.1 \%)$ in males versus $(29.1 \%)$ in females with statistically insignificant difference $(\mathrm{P}=0.666)$.

Table 6. Comparison between males and females students regarding father's parenting styles $(n=446)$.

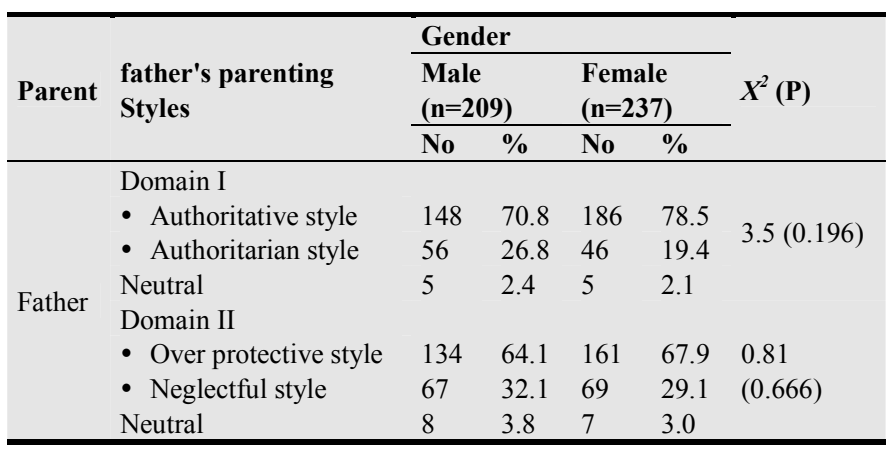

$* \mathrm{P}<0.05$ (significant)

Table 7 indicates that, regarding the first domain of mother's parenting styles; females in comparison with males had more maternal authoritative style, with $(84.4 \%)$ in females versus $(77.0 \%)$ in males, whereas males in comparison with females had more maternal authoritarian style, with $(20.1 \%)$ in males versus $(14.3 \%)$ in females with statistical insignificant difference $(\mathrm{P}=0.116)$. As regards the second domain of mother's parenting styles, the same table also shows that females in comparison with males had more maternal overprotective style, with $(86.9 \%)$ in females versus $(72.7 \%)$ in males, whereas males in comparison with females had more maternal neglectful style, with $(23.9 \%)$ in males versus $(10.5 \%)$ in females with a highly statistical significant difference $(\mathrm{P}=0.001)$. 
Table 7. Comparison between males and females students regarding mother's parenting styles ( $n=446)$.

\begin{tabular}{|c|c|c|c|c|c|c|}
\hline \multirow{3}{*}{ Parent } & \multirow{3}{*}{ Mother's parenting Styles } & \multicolumn{4}{|c|}{ Gender } & \multirow{3}{*}{$X^{2}(\mathbf{P})$} \\
\hline & & \multicolumn{2}{|c|}{ Male $(n=209)$} & \multicolumn{2}{|c|}{ Female $(n=237)$} & \\
\hline & & No & $\%$ & No & $\%$ & \\
\hline \multirow{8}{*}{ Mother } & Domain I & & & & & \multirow{4}{*}{$4.3(0.116)$} \\
\hline & - Authoritative style & 161 & 77.0 & 200 & 84.4 & \\
\hline & - Authoritarian style & 42 & 20.1 & 34 & 14.3 & \\
\hline & Neutral & 6 & 2.9 & 3 & 1.3 & \\
\hline & Domain II & & & & & \multirow{4}{*}{$14.8(0.001)^{*}$} \\
\hline & - Overprotective style & 152 & 72.7 & 206 & 86.9 & \\
\hline & - Negligence style & 50 & 23.9 & 25 & 10.5 & \\
\hline & Neutral & 7 & 3.3 & 6 & 2.5 & \\
\hline
\end{tabular}

$* \mathrm{P}<0.05$ (significant)

Figure 2 shows that among the studied males students sample, social phobia is most common with maternal authoritarian style $(23.8 \%)$ followed by maternal neglectful style $(20.0 \%)$ then paternal authoritarian style $(19.6 \%)$ and paternal neglectful style $(16.4 \%)$ with statistical insignificant differences $(\mathrm{P}=0.37$ and 0.84 respectively).

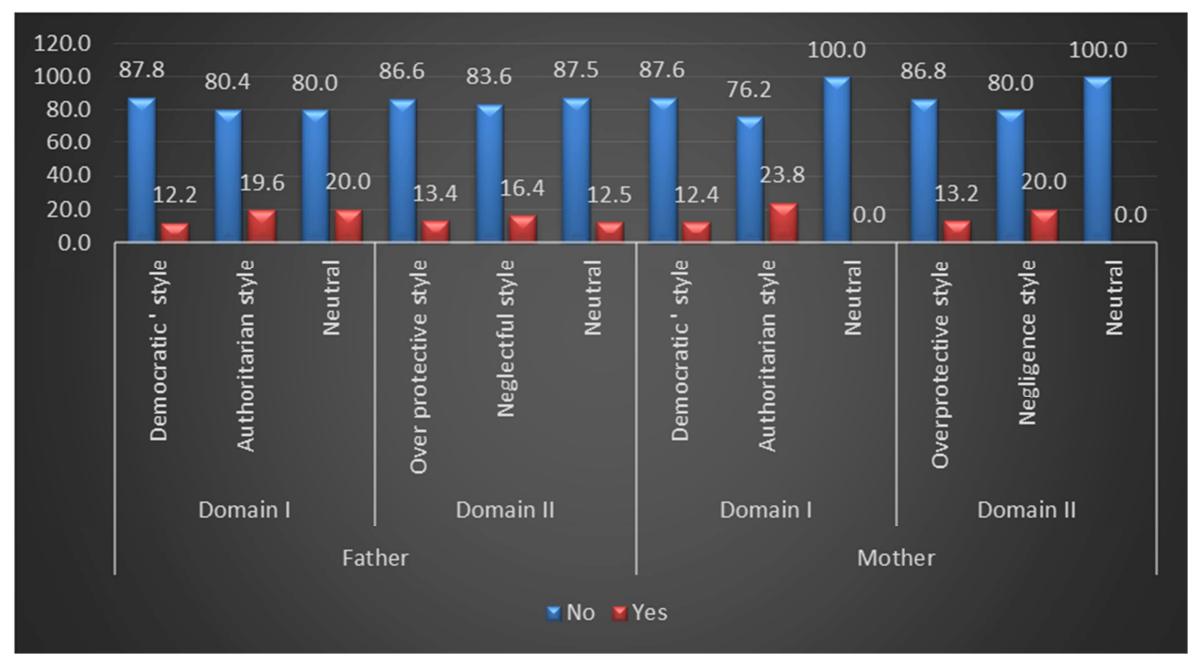

Figure 2. Distribution of social phobia among male students according to parenting styles $(n=209)$.

Figure 3 shows that among the studied female students sample, social phobia is most common with maternal neglectful style $(44.0 \%)$ with a significant difference $(\mathrm{P}=0.01)$ followed by paternal neglectful style $(39.1 \%)$ with a high significant difference $(\mathrm{P}=0.001)$ then maternal authoritarian style $(35.3 \%)$ and paternal authoritarian style $(32.6 \%)$ with statistical insignificant differences $(\mathrm{P}=0.002$ and 0.14 , respectively).

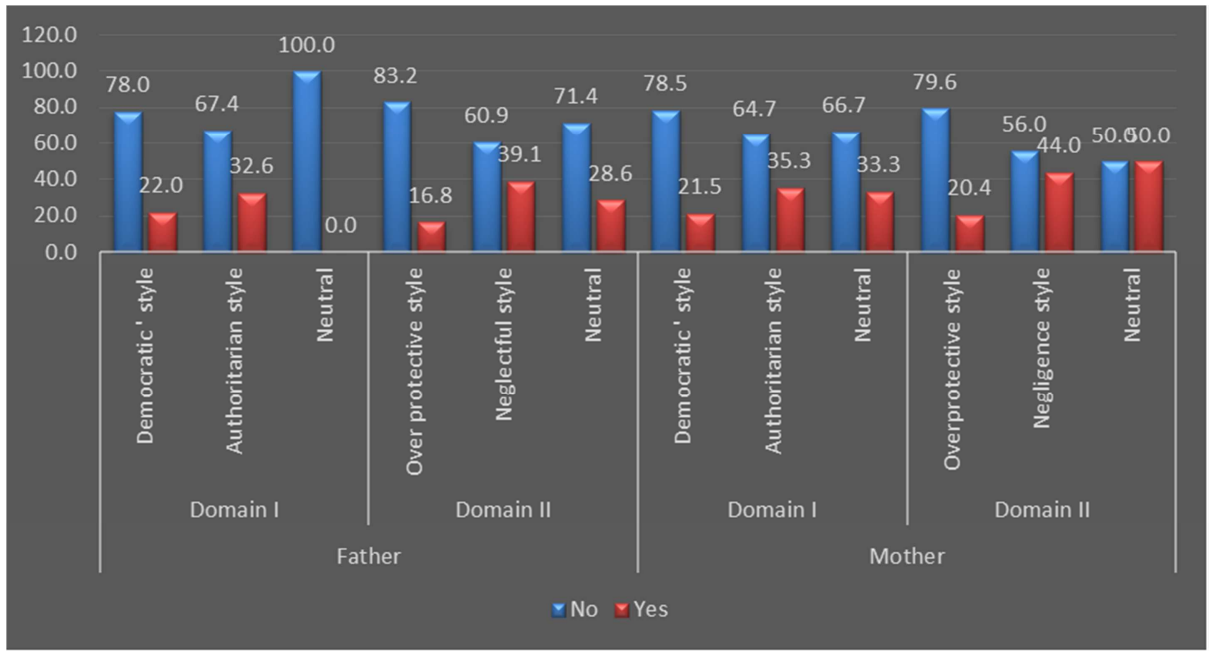

Figure 3. Distribution of social phobia among female students according to parenting styles ( $n=237)$. 
Table 8 points for that there was a statistically significant negative correlation between domain I of both paternal and maternal styles and social phobia $(\mathrm{r}=-0.10$ at $\mathrm{P}=0.041, \mathrm{r}-$ 0.11 at $\mathrm{P}=0.024$ ) respectively, and there was highly statistically significant negative correlation between domain II of both paternal and maternal styles and social phobia $(\mathrm{r}=$ 0.16 at $\mathrm{P}=0.001, \mathrm{r}=-0.11$ at $\mathrm{P}=0.016$ ) respectively.

Table 8. Correlation between parenting styles and social phobia among the studied students $(n=446)$.

\begin{tabular}{|c|c|c|c|}
\hline \multirow{2}{*}{ Variables } & \multirow{2}{*}{ Parenting styles } & \multicolumn{2}{|c|}{ Social phobia } \\
\hline & & $\mathbf{R}$ & $\mathbf{P}$ \\
\hline \multirow{2}{*}{$\begin{array}{l}\text { Paternal styles } \\
\text { "father" }\end{array}$} & $\begin{array}{l}\text { Domain I: } \\
\text { - Authoritative style } \\
\text { - Authoritarian style }\end{array}$ & -0.10 & $0.041^{*}$ \\
\hline & - Overprotective style & -0.16 & $0.001 *$ \\
\hline \multirow{2}{*}{$\begin{array}{l}\text { Maternal styles } \\
\text { "mother" }\end{array}$} & $\begin{array}{l}\text { Domain I: } \\
\text { - Authoritative style } \\
\text { - Authoritarian style }\end{array}$ & -0.11 & $0.024 *$ \\
\hline & $\begin{array}{l}\text { Domain II: } \\
\text { - Overprotective style } \\
\text { - Neglectful style }\end{array}$ & -0.11 & $0.016^{*}$ \\
\hline
\end{tabular}

r: Pearson correlation coefficient $* \mathrm{P}<0.05$ (significant)

\section{Discussion}

The quality of parenting styles represents a remarkable factor in the social, psychological and mental health development of children later in the life [23]. Anxiety disorders appear among the most common psychiatric problems, which relate to parenting styles, and are problematic for many youths throughout childhood and adolescence [24]. Specifically, social phobia is associated with severe impairments in nearly all domains of the patient's life, making it the fifth most disabling psychiatric disorders [25]. As for the sociodemographic characteristics of the studied students, the current study's result showed that the mean age of the students was $16 \pm .1$ years in both female and male students. This finding agrees with [26] who revealed in a Finnish study at University of Tampere, that the mean age in their studied adolescent sample was $15.8 \pm 1.1$ years. On contrary to this finding, did Turkish study [27], in Adnan Menderes University showed that the mean age of the studied sample was $21.16 \pm 1.76$ years. This finding might be due to transition to secondary school stage and the need for mental adjustment to pubertal body changes. As regards, the gender difference in social phobia is concerned; the present study's result clearly showed that female students exhibited a higher prevalence of social phobia than did male students with statistically significant differences. This result was congruent with [28] who found in an Iraqi study that the rate of social phobia among female students was greater than did in male students at $\mathrm{Al}$ Qadissia Medical College in Al- Dwainia city. On the contrary, with this finding a Jordanian study done by [29] revealed that there was no statically difference in social phobia among students of Isra University on the base of gender. This finding might due to earlier pubertal development in girls than in boys and associated advanced noticeable physical changes that can cause body dissatisfaction and unfavorable sexual attention, which are thought to be more prone to negative evaluation and sensitive to other's opinion regarding their appearance. Regarding the ages' onset of social phobia among students, the current study's result showed that social phobia had an earlier onset in females than did in males. This result contrasts with an American survey done by [30] who stated that there was no gender differences observed in the age of onset of social phobia. This finding might be due to the eastern society impose strict traditions on girls just reaching the pubertal age, which force them to avoid several social situations that will lead to a great number of social fears and fear of social or performance situation. As regards the parents' marital status and social phobia, the current study result revealed that males and female students coming from divorced and widowed homes were more prone to develop social phobia than those from married homes. This result is consistent with [31] who reported in a Nigerian study that parental separation in terms of divorce has a significant influence on adolescent's social phobia. While, a German study [32] showed that the increased risk for phobia was associated with parental death, not with parental separation. This occurrence could be due to divorcing or loss of parents are considered to be traumatic life events that threaten the adolescents' development toward individuation, losing security of parents' attachment and feeling as being neglected from their parents and. Regarding residence and social phobia, the rate of social phobia in male students was higher in urban slum areas with statistically significant difference, while this rate in female students was higher in rural areas without a significant difference. This finding was similar to an Egyptian study [33] among undergraduate adolescent students of Al Azhar University, where the rate of social phobia was higher among rural areas' students than urban and suburban' students. Unlikely, this result was incongruent with an Egyptian prospective study done in outpatient clinics at Mansoura University Hospital [34] revealed that most of socially phobic males and females were from urban areas. This finding might be due to the lowest living standards in urban slum areas that affect negatively on children's educational level, and decreasing the opportunities of acquiring appropriate social skills for social situations. As well, the culture in rural areas has an influential role in the presentation of that disorder. Namely, parents and environmental habits usually limit exposure to social situations. Concerning, differences in domain I of parenting styles based on the student's gender, parents exhibit more authoritarian parenting style with boys than with girls and exhibit a more authoritative parenting style with girls than with boys. Furthermore, mothers were more likely than fathers to use an authoritative style, whereas 
fathers were more likely than mothers to use an authoritarian parenting style were. In the same line of this finding, it was revealed in an Iranian study [35] among adolescents that boys in comparison with girls, their parents are more authoritarian. In addition, American study [36] that girls in comparison to boys perceive their fathers to be more authoritative towards them. This finding owned to the traditional punitive role of fathers that was inherited from their parents. Regarding differences in domain II of parenting styles based on the student's gender, parents were more likely to use neglectful style when parenting sons, whereas over-protective style was more likely to be used when parenting daughters. Furthermore, mothers significantly were more likely than fathers to use an overprotective parenting style, whereas fathers were more likely than mothers to use a neglectful parenting style. This result goes along with Indian study [37] which applied on high school students that girls significantly outnumbered boys in the high maternal protection group, while there were more boys than girls in the low maternal protection group. However, this finding contradicts with Albanian study [38] that was conducted in public schools in Tirana, revealed that the negligent parenting style is more addressed to girls than to boys. This finding could be owned to parents' assuming that boys are stronger and can defend themselves better than girls who could be easily hurt and become in danger.

Concerning the relation between domain I of parenting styles and social phobia, this study showed that authoritarian parenting style was related to social phobia among both males and female students with no significant difference. This finding is in line with a Pakistani study [39] which indicated that parents' authoritarian behavior is a source of social anxiety in adolescents.. This finding might due to strict, harsh discipline and physical punishment of authoritarian parents in upbringing their children who would develop feelings of losing the control over situations involving them, besides having poor social skills. The present study also revealed that, social phobia was statistically significant inverse correlated to authoritarian paternal and maternal style. Namely, more the parents showed authoritarian in their parenting style, their children showed lower levels of social phobia. Unlike this finding, [40]-[41] a Pakistani study and Iranian study, respectively, mentioned that social phobia was positively correlated with authoritarian parenting style. This finding might be argued that, adolescents perceived authoritarian parenting as an instrumental rigorous method for behavioral regulation and enforcing appropriate social behavior, not dictatorial or hostile control. Additionally, the current study showed that, social phobia was statistically significant inverse correlated to authoritative paternal and maternal style. It meant that more the parents showed authoritative in their parenting style, their children showed lower levels of social phobia. Consistent with this finding, Indian study [42] reported that authoritative parenting style was negatively correlated with social phobia. On contrary to this finding, a Pakistani study [40] mentioned that social phobia in girls was positively correlated with authoritative parenting style. This finding could be owned to authoritative parents encourage effective communication and closed relationship with their children that would positively reflect on children's psychosocial de Regarding relation between domain II of parenting styles and social phobia, this study also revealed that paternal and maternal neglectful parenting style were associated with social phobia among both males and female students with a highly significant difference in the females. On the same line of this finding, American study [43] revealed that a significant differences were found for social anxiety scores by maternal neglectful parenting style. This finding might due to parents spend most of their time at work for saving physical needs for their children, and so they become less involved with their children. The current study also demonstrated that, social phobia was statistically significant inverse correlated to neglectful paternal and maternal style. Our finding is consistent with American study [44] who documented the detrimental effect of neglectful parenting on children's psychological health as they become less psychosocial mature, less competent and more trouble. On contrary to this finding, Indian study [42] showed that neglectful parental style was positively correlated with social phobia. This finding could be speculated that since the adolescents do not receive necessary care and attention or gaining proximity of their parents due to busyness with their works, children often become independent and develop appropriate social interactional skills with others, thereby, any control exerted on those children would negatively affect and conflict with their social relations. Moreover, our study revealed that, social phobia was statistically significant inverse correlated to overprotective paternal and maternal style. This finding is in line with Romanian study [45] who reported that there was statistically significant negative correlations between child's social phobia and paternal overprotection. However, this finding contradicts with an American study [46] revealed that there were significant positive correlation between social phobia and Maternal Overprotection and Paternal Overprotection. This finding might be owned to overprotection exerted over children diminish their ability of procuration the necessary social skills independently, thereby become feeling with loss of control at perceiving social situations.

\section{Conclusion}

Authoritarian and neglectful parenting styles are more likely to cause social phobia among the adolescents.

\section{Recommendation}

Health educational programs for parents about the effective style in dealing and parenting their children. Further research to study the relation between parenting styles and social development in adolescents. 


\section{References}

[1] Powell VB, Oliveira OH, Seixas C, Almeida C, Grangeon MC, Caldas M, Bonfim TD, Castro M, Galvão-de Almeida A, Moraes RO, Sudak D \& de-Oliveira IR. (2013): Changing core beliefs with trial-based therapy may improve quality of life in social phobia: a randomized study. Revista Brasileira de Psiquiatria; 35 (3): 243-247.

[2] American Psychiatric Association (2013): Diagnostic and statistical manual of mental disorders (Fifth Edition (DSM-5). Washington.

[3] American Psychiatric Association. (2000): Diagnostic and statistical manual of mental disorders (4th ed-TR). Washington, DC: Author.

[4] Kerns CE, Comer JS, Pincus DB \& Hofmann SG. (2013): Evaluation of the Proposed Social Anxiety Disorder Specifier Change for Dsm-5 in a Treatment-Seeking Sample of Anxious Youth. National Institute of Health Public Access; 30 (8): 709715 .

[5] Bögels SM, Alden L, Beidel DC, Clark LA, Pine DS, Stein MB \& Voncken M. (2010): Social anxiety disorder: Questions and answers for the DSM-5. Depression and Anxiety; 27 (2): 168-189.

[6] Merikangas KR, He JP, Burstein M, Swendsen J, Avenevoli S, Case B \& Olfson M. (2011):Service utilization for lifetime mental disorders in U.S. adolescents: Results of the National Comorbidity Survey - Adolescent Supplement (NCS-A). Journal of the American Academy of Child and Adolescent Psychiatry; 50 (1): 32-45.

[7] Gallagher M, Prinstein MJ, Simon V \& Spirito A. (2014): Social Anxiety Symptoms and Suicidal Ideation in a Clinical Sample of Early Adolescents: Examining Loneliness and Social Support as Longitudinal Mediators. J Abnorm Child Psychol; 42 (6): 871-883.

[8] verach L \& Rapee RM. (2014): Social anxiety disorder and stuttering: Status and future directions. Journal of Fluency Disorder;. 40: 69-82.

[9] Knappe S, Beesdo K \& Pine DS. (2009): Anxiety and anxiety disorders in children and adolescents: developmental issues and implications for DSM-V". Psychiatr Clin North Am; 32 (3): 483-524.

[10] Kordi A \& Baharudin R. (2010): Parenting Attitude, Style, and Its Effect on Children's School Achievements. International Journal of Psychological Studies; 2 (2): 217-222.

[11] Lokoyi OLO. (2015): Parenting Styles as Correlates of Aggressive Behavior among In-School Adolescent with Mild Intellectual Disability. Psychology and Behavioral Sciences; 4 (3) 94-100.

[12] Tunde-Ayinmode MF \& Adegunloye OA. (2011): Parenting style and conduct problems in children: A report of deliberate selfpoisoning in a Nigerian child. Department of Behavioural Sciences, University of Ilorin Teaching Hospital, Ilorin, Nigeria; 17 (2): 60-63.

[13] Pong S-L, Johnston J \& Chen V. (2010): Authoritarian Parenting and Asian Adolescent School Performance: Insights from the US and Taiwan. Int J Behav Dev; 34 (1): 62-72.
[14] Massachusetts General Hospital, School Psychiatry Program \& MADI Resource Centre (2010): school based intervention of social anxiety: School Psychiatry Program and MADI Resource Centre. Retrieved 7 October 2015, from http://www2.massgeneral.org/.

[15] Ryan JL \& Warner CM. (2012): Treating Adolescents with Social Anxiety Disorder in Schools. Child Adolesc Psychiatr Clin N Am; 21 (1): 105-118.

[16] Bolsoni-Silva AT \& Loureiro SR. (2014): The Role of Social Skills in Social Anxiety of University Students. Paideia; 24 (58): 224.

[17] Chan TW \& Koo A. (2011): Parenting style and youth outcomes in the UK. European Sociological Review; 27 (3): 385-399.

[18] Nikoogoftar M \& Seghatoleslam S. (2015): The Role of Parenting Styles in Predicting Adolescent Behavioral and Emotional Problems. Research Papers; 3 (1): 23-30.

[19] El-Gilany A, El-Wehady \& El-Wasify M. (2012): Updating and validation of the socioeconomic status scale for health research in Egypt. Eastern Mediterranean Health Journal; 18 (9): 962-968.

[20] Sakkar E. (1984): Anmatt Eltanshea Elwaldya Waelaqetaha Belkhagl Lada Talba Elsaf Elawl Elthanwy Fi Mohafaza Ram -Allah W Elbeila. Al-Quodss University. Palstine.

[21] Raulin ML \& Wee JL. (1994): Social fear scale. In J. Fisher \& K. Corcoran (Eds.), Measures for clinical practice: Asource book second ed. 2:595. New York: The free press.

[22] El-Desoky M. (2004): Arabic version of Social phobia scale. Ed. by Alnhda library Cairo-Egypt.

[23] Otowa T, Gardner CO, Kendler KS \& Hettema JM. (2013): Parenting and risk for mood, anxiety and substance use disorders: a study in population-based male twins. Soc Psychiatry Psychiatr Epidemiol; 48 (11): 1841-1849.

[24] Saad K, Al-Atram AA, Abdel Baseer KA, Ali AM \& ElHoufey AA (2015): Assessment of Quality of life, Anxiety and Depression in Children with Turner syndrome: A CaseControl Study. American Journal of Neuroscience; 6: 8.12.

[25] Jazaieri H, Morrison AS, Goldin PR \& Gross JJ. (2015): The Role of Emotion and Emotion Regulation in Social Anxiety Disorder. Curr Psychiatry Rep; 17 (531):1-9.

[26] Ranta K, Kaltiala-Heino R, Pelkonen M \& Marttunen M. (2009): Associations between peer victimization, self-reported depression and social phobia among adolescents: the role of comorbidity. Journal of Adolescence; 32 (1): 77-93.

[27] Gültekin BK \& Dereboy I F. (2011): The prevalence of social phobia, and its impact on quality of life, academic achievement, and identity formation in university students. Turkish Journal of Psychiatry; 22 (3): 150-158.

[28] Alkhafji AZM. (2012): Social phobia among Al Qadissya medical student: prevalence, Academic performance and response to different treatments; 54 (1): 33-37.

[29] Alhabees FA \& Alkhutaba MY. (2015): Social phobia among Isra University students in light of some variables. Global Journal of Psychology and Behavioural Education; 3 (2): 088091. 
[30] McLean CP, Asnaani A, Litz BT \& Hofmann SG. (2011): Gender Differences in Anxiety Disorders: Prevalence, Course of Illness, Comorbidity and Burden of Illness. Journal of psychiatric research; 45 (8): 1027-1035.

[31] Uzonwanne FC \& Akpunne BC. (2015): Influence of Family Status and Relationships on Social Phobia among Young Adults in Nigerian Universities. Advances in Social Sciences Research Journal; 2 (1): 11-23.

[32] Beesdo K, Knappe S \& Daniel S \& Pine DS. (2009): Anxiety and anxiety Disorders in children and adolescents: Developmental issues and implications for DSMV. The Psychiatric clinics of North America; 32 (3): 483-524.

[33] Ragheb K, Abd El-Azem S, Abdel-wahab M, Attia H, Ismail R, Said M \& Taha H. (2009): Prevalence of Social Anxiety Symptoms Among a Sample of Egyptian Adolescents. Egyptian Journal of Psychiatry; 29 (1): 5-13.

[34] Amr M, El-Wasify M, El-Gilany A-H \& Rees S. (2013): Gender differences among patients with social phobia in Egypt. The Arab Journal of Psychiatry; 24 (1): 52-59.

[35] Zeinali A. (2014): Relationship of Parenting Style with SelfRegulation in Adolescent. Journal of Applied Science and Agriculture; 9 (3): 1105-1121.

[36] Shalini A \& Acharya YT. (2013): Perceived Paternal Parenting style on Emotional Intelligence of Adolescents. Guru Journal of Behavioral and Social Sciences; 1 (4): 194202.

[37] Dash M \& Sriranjan P. (2014): Maternal Over-Protection and Achievement Motivation among High School Students. Journal of Humanities and Social Science; 19 (5): 57-62.

[38] Kashahu L, Dibra G, Osmanaga F \& Bushati J. (2014): The relationship between parental demographics, parenting styles and student academic achievement. European Scientific Journal; 10 (13): 237-251.

[39] Akhtar S, Rana SA \& Tahir MA. (2013): Parenting Styles and Social Anxiety among Adolescents. New Horizons; 7 (2): 115.

[40] Yousaf S. (2015): The Relation between Self-esteem, Parenting Style and Social Anxiety in Girls. Journal of Education and Practice; 6 (1): 140-142.

[41] Shabnam B, Shaeeri MR \& Mansour HJ. (2010): Social Phobia, Parenting Styles, and Perfectionism. Developmental Psychology (Journal of Iranian Psychologists); 7 (25): 75-83.

[42] Sandhu GK \& Sharma V. (2015): Social Withdrawal and Social Anxiety in Relation to Stylistic Parenting Dimensions in the Indian Cultural Context. Research in Psychology and Behavioral Sciences; 3 (3): 51-59.

[43] Anhalt K \& Morris TL. (2008): Parenting Characteristics Associated With Anxiety And Depression: A Multivariate Approach. Journal of Early and Intensive Behavior Intervention; 5 (3): 122-137.

[44] Steinberg L, Eisengart IB \& Caufmann E (2006): Patterns of competence and adjustment from Authoritative, Authoritarian, Indulgent and Neglectful homes: A replication in a sample of serious juvenile Offenders. Journal of Research on Adolescence; 16 (1): 47-58.

[45] Dobre C, Rădulescu D, Gabor S, Gherasim AM \& Vas R. (2014): Parenting style and child anxiety. Romanian Journal of Cognitive Behavioral Therapy and Hypnosis; 1 (4): 1-13.

[46] Spokas M \& Heimberg RG (2009): Overprotective Parenting, Social Anxiety, and External Locus of Control: Crosssectional and Longitudinal Relationships. Cognitive Therapy \& Research; 33 (6): 543-551. 